\title{
Economic Dispatch Model Based on Time-of-Use Electricity Price for Photovoltaic Systems
}

\author{
Zhifeng Zhong $^{1} \cdot$ Yi Zhang $^{1} \cdot$ Shihui Wang ${ }^{1} \cdot$ Chengge Deng $^{1} \cdot$ Yan Zhang $^{2}$ (I)
}

Received: 23 May 2017 / Accepted: 16 November 2017 / Published online: 20 December 2017

(C) The Author(s) 2017. This article is an open access publication

\begin{abstract}
Due to the energy storage limitations of photovoltaic systems and the uneven time-of-use of electricity by users and the policies of different time-of-use electricity price, scheduling and dispatching of photovoltaic systems incurs irregular trend of electricity consumption and associated price hikes which influences by supply and demand. To this end, this paper proposes a novel model for photovoltaic system based on sensor networks system exploiting the historical factual data and the real-time data obtained from various sensors of the system, where adaptive particle swarm optimization and bacteria foraging algorithms are used for optimizing the scheduling process. The algorithm enhances the capacity of both global and local searches, and the convergence speed and estimation accuracy are promoted obviously compared to the other algorithm. Experiments results demonstrate that the ED model in this paper has the effect of peak cutting and valley filling, which ultimately bring the greatest economic benefits to the users.
\end{abstract}

Keywords Economic dispatch · Monitor sensors systems · Time-of-use electricity price

\section{Introduction}

Given the ever increasing consumption of natural and conventional resources to facilitate supplements for human utilities and the growing awareness for energy efficiency, invention and utilization of alternative sources of energy supplements have naturally become a necessity. Recent scientific studies [1,2] have demonstrated that solar energy could

Yan Zhang

2315918@qq.com; zhangyan@hubu.edu.cn

Zhifeng Zhong

zfzhong@hubu.edu.cn

Yi Zhang

1160468643@qq.com

Shihui Wang

631793307@qq.com

Chengge Deng

1358235647@qq.com

1 School of Computer Science and Information Engineering, Hubei University, Wuhan 430062, Hubei Province,

People's Republic of China

2 Education Information Engineering Center of the School of Computer Science and Information Engineering, Hubei University, Wuhan 430062, Hubei Province, People's Republic of China potentially be a promising source of natural energy with the capacity of availing abundant supplements for human living in the future. However, due to the rapid growth of power demands and the users' uneven time-of-use electricity, usage patterns of the electricity grid are characterizing increased fluctuations along with associated increase in the load rate [3]. In order to reduce the load rate of the power grids, grid-connected operation of large-scale distributed photovoltaic systems is being adopted. Due to the highly random and fluctuating nature of photovoltaic power generation, this operation scheme would inevitably introduce distributions to the current power grids. Consequently, it is important to investigate the scheduling mechanisms for photovoltaic power generation. Time-of-use electricity price (TOUEP) is a policy which draws important guidelines for electricity demands and power supply-related managements and regulations for international power industries. TOUEP has been proven to reduce load rates by price element. The price is expensive during peak time, which can reduce the electricity demand. On the contrary, the price is cheap during valley time, which can increase electricity demand. That can be reached to balance of supply and demand to a certain degree, and then improve the reliability of power grids and reduce the user expenditures by changing the time-of-use electricity power reasonable [4]. Furthermore, the TOUEP-based eco- 
nomic dispatching (ED) of photovoltaic power systems is expected to be an integral component of power industries in the future.

A wide range of research works have been proposed to date in the context of ED in power grids. The work presented in [5] introduced a mathematical model that considers both generation cost and emission cost, and proposes a solution based on the chaotic ant swarm algorithm. Similarly, the works of [6] introduced a model that utilizes the forecasting information of power generation time slots of wind and the power generations of the photovoltaic system for the purpose of planning the power generation and interruptible loads for the spinning reserve. This model has been developed based on the improved discrete particle swarm and continuous particle swarm algorithms. Further, a chaotic quantum genetic algorithm-based model has been proposed in [7] considering the operational and pollution emission costs. A TOUEP-based ED model has been proposed in [3] focusing on the operational cost on photovoltaic power generation with an analytical approach using particle swarm algorithm. The works of [8] generated an optimized micro-power grid dispatching model based on an improved bacteria foraging algorithm, focusing on the meteorological conditions of power grid construction location, investment, operational and maintenance costs. In order to maximize the overall benefits, a power dispatching model considering the operational and pollution emission costs of a micro-power grid has been proposed in [9], based on a combination of Monte Carlo simulation and particle swarm algorithm. Four different types of power dispatching problems in power grid systems, such as multi-area ED with tie line limits, ED with multiple fuel options, combined environmental ED and the ED of generators with prohibited operating zones, have been investigated and solved via a particle swarm algorithm in [10]. Two power testing models considering the operational and pollution emission costs of power systems have been established in [11]. Again, a multi-objective chaotic particle swarm algorithm has been used to find the solution. The works of [12] used Lbest-PSO with dynamically varying subswarms to address the dynamic economic dispatch problem. The aim of this work is to minimize the cost of power production of all the participating generators over a $24 \mathrm{~h}$ time period. An improved firefly algorithm [13] has been proposed to solve ED model by considering the prohibited operation zones and ramp rate limits. Similarly, an efficient stochastic multi-period dynamic ED model has been proposed [14] by utilizing a mixed Gaussian distribution.

Compared with the literature [5-8] and [10-14], this paper has given enough considerations to the influence of TOUEP on ED and comprehensively considered grid-connected operation that better than the literature [8] which only discuss the micro-power generation system. In addition, the algorithms using in this paper avoid the prematurity and obtain high accuracy from the convergence which is better than that in [3] and [9].

To alleviate these issues, this paper proposes a scheduling model that deals with the ED problem of photovoltaic power systems under grid-connected and off-grid modes, and it is particularly designed for the household business type of photovoltaic power stations. In general photovoltaic power stations provide historical information of power generation, which are usually stored in the connected networks under grid-connected mode and in the stand-alone system under off-grid mode of operation, respectively. All such data are usually collected by a number of environmental sensors, which include measurement of temperature, humidity, light intensity and voltage.

This paper proposes a daily basis ED model with the primary objective of maximizing the operational profit while considering several factors including maximal output of battery and photovoltaic cell arrays, the constraint of maximal interactive power between photovoltaic systems and power grids as well as the characteristic of battery power storage. Furthermore, a novel TOUEP-based self-adaptive particle swarm optimization-bacteria foraging optimization (APSOBFA) algorithm has been developed to achieve the objectives of our proposed model. Experimental analysis demonstrates the fast convergence and highly accurate estimation capabilities of our proposed model for achieving effective daily power dispatching in terms of both global and local searching. Moreover, our proposed model converges in 20 iteration cycles to yield the optimal result, thereby achieving timely power dispatching and ease of use from engineering perspectives.

The remainder of this paper is organized as follows: Sect. 2 introduces the proposed model of ED for photovoltaic power systems, and Sect. 3 details our hybrid optimization algorithm. Section 4 discusses the experimental analysis and results, and Sect. 5 concludes this paper.

\section{The Model of ED for Photovoltaic Power Generation Systems}

\subsection{Objective Function}

The operational costs of photovoltaic power generation systems mainly consist of the power generation cost and the interaction cost between photovoltaic systems and existing power grids when the PV-generated electricity is insufficient which needed to purchase from the power grids. The power generation cost includes the maintenance cost of batteries and photovoltaic cells, while the interaction cost includes the cost of buying electricity from power grids and profit generated by selling electricity to power grids. Under the current policy of TOUEP, the electricity price varies under different time slots. 
Therefore, it beneficial to derive a daily dispatching plan for the generated photovoltaic power from the user perspective. This paper establishes an ED model based on TOUEP for photovoltaic power generation systems. The maximal operational profit is defined as the objective function given by:

$$
\begin{aligned}
C_{\mathrm{max}}= & \sum_{i=1}^{T}\left(C_{\mathrm{sel}, t} P_{\mathrm{sel}, t}-C_{\mathrm{buy}, t} P_{\mathrm{buy}, t}-C_{\mathrm{pv}} P_{\mathrm{pv}, t}\right. \\
& \left.-C_{\mathrm{bat}}\left|P_{\mathrm{bat}, t}\right|\right)
\end{aligned}
$$

where $C_{\max }$ is the operation profit of photovoltaic systems, $t$ is the dispatching time slot, $T$ is the maximum time slot, $C_{\text {sel, } t}$ and $C_{\text {buy, } t}$ are electricity sale and purchase prices, respectively. $P_{\mathrm{sel}, t}$ and $P_{\mathrm{buy}, t}$ are the numbers of electricity powers sold and bought, respectively. $C_{\mathrm{pv}}$ and $C_{\text {bat }}$ are the maintenance costs of photovoltaic cells and storage per battery. $P_{\mathrm{pv}, t}$ and $P_{\mathrm{bat}, t}$ represent the generation power of the photovoltaic cells and charge-discharge power of batteries of time slot $t$.

\subsection{Constraint Conditions}

The constraint conditions of photovoltaic systems include systematic power balance which mean that input and output of the power in system no matter what ways are equivalent during every dispatching time slot, maximum battery output power, state of charge (SOC) of batteries and maximal mutual power transmission between photovoltaic systems and power grids.

\subsubsection{System Power Balance Constraints}

$P_{\mathrm{buy}, t}+P_{\mathrm{pv}, t}+P_{\mathrm{bat}, t}=P_{\mathrm{sel}, t}+P_{\mathrm{load}, t}$

where $P_{\text {load }, t}$ is the power consumer use. The other parameters are the same as Eq. (1). If the conversion efficiency of the inverter and the charge-discharge power of a storage battery is considered in the actual situation, then Eq. 2 could be written as follows:

$P_{\mathrm{buy}, t}+P_{\mathrm{pv}, t} \eta_{\mathrm{pv}}+P_{\mathrm{bat}, t} \eta_{\mathrm{bat}}=P_{\mathrm{sel}, t}+P_{\mathrm{load}, t}$

Where $\eta_{\mathrm{pv}}$ is the conversion efficiency of the inverter, $\eta_{\mathrm{bat}}$ is the charge-discharge power of the storage battery.

\subsubsection{Output of the Power Constraints of Battery}

$$
P_{\text {batmin }} \leq\left|P_{\text {bat }, t}\right| \leq P_{\text {batmax }}
$$

where $P_{\text {batmin }}$ and, $P_{\text {batmax }}$ represent the lower and upper bounds of battery output power, respectively.

\subsubsection{Battery SOC Constraints}

$\mathrm{SOC}_{\min } \leq \mathrm{SOC}_{t} \leq \mathrm{SOC}_{\max }$

where $\mathrm{SOC}_{\min }$ is the lower bound of battery $\mathrm{SOC}, \mathrm{SOC}_{\max }$ is the upper bound of battery SOC.

\subsubsection{Interval Power Constraints}

$\left\{\begin{array}{l}0 \leq P_{\text {sel }, t} \leq P_{\text {selmax }} \\ 0 \leq P_{\text {buy }, t} \leq P_{\text {buymax }}\end{array}\right.$

where $P_{\text {selmax }}$ is the upper bound of selling power and $P_{\text {buymax }}$ is the upper bound of buying power during dispatching time slot.

\section{Hybrid Optimization Algorithm}

\subsection{Particle Swarm Optimization (PSO)}

The particle swarm optimization algorithm was proposed by Kennedy and Eberhart in 1995 [15,16]. It is a bionic global optimization algorithm developed based on the strategy of searching food for a bird flock. The algorithm treats the problem search space as the food search space of the bird's flock and assumes every bird as a particle which can be considered as a candidate solution to the solution space. For PSO, the position and velocity of the particle should be firstly initialized and then the swarm optimal solution should be searched by tracking the two extreme values [3]. The first extreme value is an optimal solution according to a particle's own experience, and the second is an optimal solution according to the total swarm's experience $[17,18]$.

Let the swarm size be $s$, the dimension be $d$, then the position of the particle is $X=\left(x_{i, 1}, x_{i, 2}, \ldots, x_{i, d}\right)^{T}$, and the velocity is $V=\left(v_{i, 1}, v_{i, 2}, \ldots, v_{i, d}\right)^{T}$, where $x_{i, d}$ is the position along the dimension $d$ of particle $i, v_{i, d}$ is the velocity along dimension $d$ of particle $i$.

The velocity and position are updated using the following formula:

$$
\left\{\begin{array}{l}
x_{i}^{j+1}=x_{i, d}^{j}+x_{i, d}^{j+1} \\
v_{i, d}^{j+1}=w v_{i, d}^{j}+c_{1} \text { rand }_{1}^{i}\left(x_{i, d}^{j}-\text { xgbest }_{i}\right) \\
+c_{2} \text { rand }_{2}^{i}\left(x_{i, d}^{j}-x_{z b e s t}\right)
\end{array}\right.
$$

where $i$ is the particle $(1 \leq i \leq s), j$ is the iterations, $\omega$ is the inertia constant, rand $_{1}^{i}$ and rand $_{2}^{i}$ are two independently and uniformly distributed random variables with range $[0$, $1]$, and $c_{1}, c_{2}$ are particle learning factor and swarm learning factor, respectively $[19,20]$.

PSO benefits from a strong global searching capability and is able to find the neighborhood containing the optimal 
value within a limited time constraint. However, it likely suffers from the weakness and premature convergence problems during the local searching process.

\subsection{Bacterial Foraging Algorithm (BFA)}

The bacterial foraging algorithm was proposed by Liu and Passino [21], which is a bionic optimization algorithm for simulating the foraging behavior of Escherichia coli in the human intestinal tract. This algorithm employs a strategy of retaining the bacteria with stronger feeding ability and discarding the weaker ones. It includes three main steps [22, 23]:

\subsubsection{Chemokines Operation}

The action of moving to a high-nutrient zone is known as chemotaxis. The chemokines operation includes tumbling and running.

The equation for the tumbling process is given as follows:

$$
\left\{\begin{array}{l}
P(i, m+1, k, l)=P(i, m, k, l)+v(i) \emptyset(i) \\
\emptyset(i)=\frac{\Delta(i)}{\sqrt{\Delta^{T}(i) \Delta(i)}}
\end{array}\right.
$$

The equation for the moving process is given as follows:

$$
P(i, m+1, k, l)=P(i, m, k, l)+v(i)
$$

where $P(i, m, k, l)$ is the position of the $i$ th bacteria at the $m$ th chemokine, the $k$ thbreeding and the $l$ th disperse, respectively, $v(i)$ is the step size of the running operation, $\varnothing(i)$ is the selected direction vector of the tumbling process, and $\Delta(i)$ is a random vector during the process of the tumbling.

\subsubsection{Breeding Operation}

The breeding operation is implemented once the maximal number of the iterations is reached. Taking the fitting level of bacteria as the criterion, half of the stronger bacteria are retained and the other half discarded by the BFA. Subsequently, the remaining bacteria are divided into two subgroups with identical positions and step length.

The equation of the breeding operation is as follows:

$$
\left\{\begin{array}{l}
P(2 i, m, k, l)=P(i, m, k, l) \quad(0 \leq i \leq s / 2) \\
v(2 i)=v(i) \quad(0 \leq i \leq s / 2)
\end{array}\right.
$$

where $P$ is the new swarm after completion of the sorting operation. It uses the retained bacteria with better status to replace the poor bacteria, and the corresponding step size will be eliminated and replaced to reform a new population.

\subsubsection{Disperse Operation}

The dispersion operation will be implemented once the maximal number of breeding is achieved. Let us select a proportion of bacteria with a certain probability of breading and disperse them to the searching space.

Now, the BFA has a good local searching capability due to the Chemokines operation. The breeding operation ensures the population diversity of BFA, and the dispersing operation enhances the global search searching capability of BFA.

\subsection{APSO-BFA}

This section introduces our proposed novel APSO-BFA algorithm based on the conventional PSO algorithm and the BFA algorithm. This algorithm optimizes both the local searching process by utilizing chemokines operation and the global searching process by adding breeding and dispersion operations. In addition, an adaptive operator is incorporated into the running step of chemokines. The combination of APSO-BFA benefits from strong global search capability, driven by PSO and local searching capability of BFA.

The equation of the adaptive step is as follows:

$\omega(k+l)=\omega_{\max }-\left(\omega_{\max }-\omega_{\min }\right)\left(\frac{k+l}{k_{\max }+l_{\max }}\right)^{2}$

where $\omega_{\max }$ and $\omega_{\min }$ are the minimum and maximum value of the inertia constant, respectively, $k_{\max }$ is the maximum value of the breeding operation, $l_{\max }$ is the maximum of the dispersing operation. $\omega$ changes slowly during the early stages, and this feature maintains the whole searching capability of the PSO. In later stages, $\omega$ changes rapidly which greatly improves the local searching capability. In summary, the self-adaptive step of $\omega$ retains all the benefits of PSO and BFA.

The main steps of APSO-BFA are described as follows: Step 1 Consider the relevant parameters of photovoltaic power systems including maintenance cost of batteries, electricity price, output power and maintenance cost of photovoltaic cells and power load of the systems as the entry arguments.

Step 2 Initialize the swarm by defining the swarm size $s$, the maximum number of iterations Maxit, the maximum number of dispersions $\mathrm{Ned}$, the probability of dispersion Ped, the maximum rate of breeding Nre, the maximum number of chemokines operation $m$ and the maximum number of movement $M$. At the same time, generate the swarm velocity at different random time slots, where the moving speed is equivalent to the moving step of the particle. In addition, the buying and selling power of the battery at different time slots is generated randomly, where the generated values are 
maintained within an acceptable dynamic range by applying several constraint conditions.

Step 3 At each iteration, the fitness level of each particle is calculated in a swarm. The historical swarm optimal value, $x z$ best , and the historical individual optimal value, $x_{\text {gbest }}$, are obtained by sorting all the fitness levels including the current and previous swarms.

Step 4 Update the velocity and position of each particle using Eq. (7) and its latest fitness level is then calculated. The values of fitness level before and after each update are evaluated against each other and thereafter the historical individual optimal value, xgbest $_{i}$ of each particle is obtained. Subsequently, each particle is moved by Eq. (9) and $x_{\text {gbest }} i_{i}$ is calculated for every step until the maximal number of updates is achieved. Finally, all the xgbest $_{i}$ within a current swarm are compared against the best achievements from the historical swarm, if there exist an $x$ gbest $_{i}$ better than the historical best $x z$ zbest, the optimal xzbest is achieved and updated.

Step 5 Assert if the number of chemokines operations reaches the upper bound. If this is the case, perform the breeding operation on the swarm in which opted for better half to duplicate them to satisfy the total number of particles remain unchanged.

Step 6 Assert if the number of breeding operations reaches the upper bound. If this is the case, perform the dispersion operation on the swarm. The xgbest $_{i}$ and $x z$ zest values are updated after the dispersion operation.

Step 7 Assert if the completed number of dispersion operations reaches the upper bound, then continue to the next iteration and repeat steps 3 to 7 .

Step 8 Once the maximal number of iterations is reached, the final two extreme values are obtained.

The flowchart is shown in Fig. 1.

\section{Experiment Results and Analysis}

This section exhibits the efficiencies of our proposed ED model in a household photovoltaic system that runs under both grid-connected and off-grid modes, where electricity purchased and sold from the power grid are both applied. To demonstrate the validity and strengths of the proposed ED model, $10 \mathrm{~kW}$ is assumed as the power scale of the system. It is worth to note that this model is applied on a daily basis, where $1 \mathrm{~h}$ is the minimum computational unit. The daily operational plan for power dispatch is then computed based on our proposed model.

A household photovoltaic system consists of a photovoltaic cell array, batteries, inverters, controllers, environment monitor and payload. The photovoltaic cell array is the most important component of a photovoltaic system that supplies the electricity to the household system, while the payload is the main unit to consume electricity. Batteries charge during the presence of electric current and discharge when the system needs electricity. Meanwhile, the photovoltaic system sells electricity to the power grid if it has sufficient electricity and buys electricity from the power with the power being insufficient. The architecture of the photovoltaic system is shown in Fig. 2.

The forecasted daily output power and the payload of the photovoltaic system from the historic datum are shown in Figs. 3 and 4. It can be observed from Fig. 3 that the output power of the photovoltaic system is highly associated with the solar radiation during the day, where the maximum power output is obtained at around 12:00 and the power output is declining thereafter and eventually reaches zero when there is no sunshine from 20:00 to 05:00 the following day.

The real electricity price in used in the Shandong Province of China has been applied in this analysis, and a single day has been divided into four periods as trough period, flat period, peak period and top period. The detailed day segmentation and associated electricity prices for both selling and buying for the household photovoltaic power systems are shown in Fig. 5.

MATLAB 2012a is used for the implementation and verification of the proposed model in this analysis. The parameters of the proposed model are given as follows:

The size of the swarm $s=20$. The maximum number of the iterations Maxit $=300$. The learning factors $c_{1}=c_{2}=2$. The maximum number of chemokines $m=4$. The maximum number of the iterations $M=4$. The maximum number of breeding cycles $\mathrm{Nre}=4$. The maximum number of dispersion operations Ned $=2$. The probability of dispersion Ped $=0.25$. The maximum power of selling electricity $P_{\text {selmax }}=15 \mathrm{~kW}$. The maximum power of the buying electricity $P_{\text {buymax }}=10 \mathrm{~kW}$. The maximum capacity of the battery $\mathrm{SOC}_{\max }=25 \mathrm{kWh}$. The minimum capacity of the battery $\mathrm{SOC}_{\min }=5 \mathrm{kWh}$. The maintain cost of the photovoltaic array $C_{\mathrm{pv}}=0.02 \mathrm{RMB} / \mathrm{kWh}$. The maintenance cost of the battery $C_{\text {bat }}=0.05 \mathrm{RMB} / \mathrm{kWh}$.

As it is shown in Table 1, 23:00-07:00 the following day is the trough period where the household photovoltaic system buys electricity from the power grid to satisfy its own payload and to charge the battery. There are no electricity sales during these periods; however, the power of the battery would reach the maximum value at the end of this period. The flat period is between 07:00 and 10:00, where the system payload only receives the electricity from the photovoltaic system, and the status of the battery remains the same. Since the solar radiation is not at its peak during this period, the system payload can buy electricity from the power grid. The peak period is between 10:00 and 15:00, where the solar radiation reaches its maximal value. Now the photovoltaic system receives the sufficient power inputs and is able to sell the electricity both generated and what in the battery to the power grid after satisfy its payload. The flat period is between 15:00 and 18:00, 


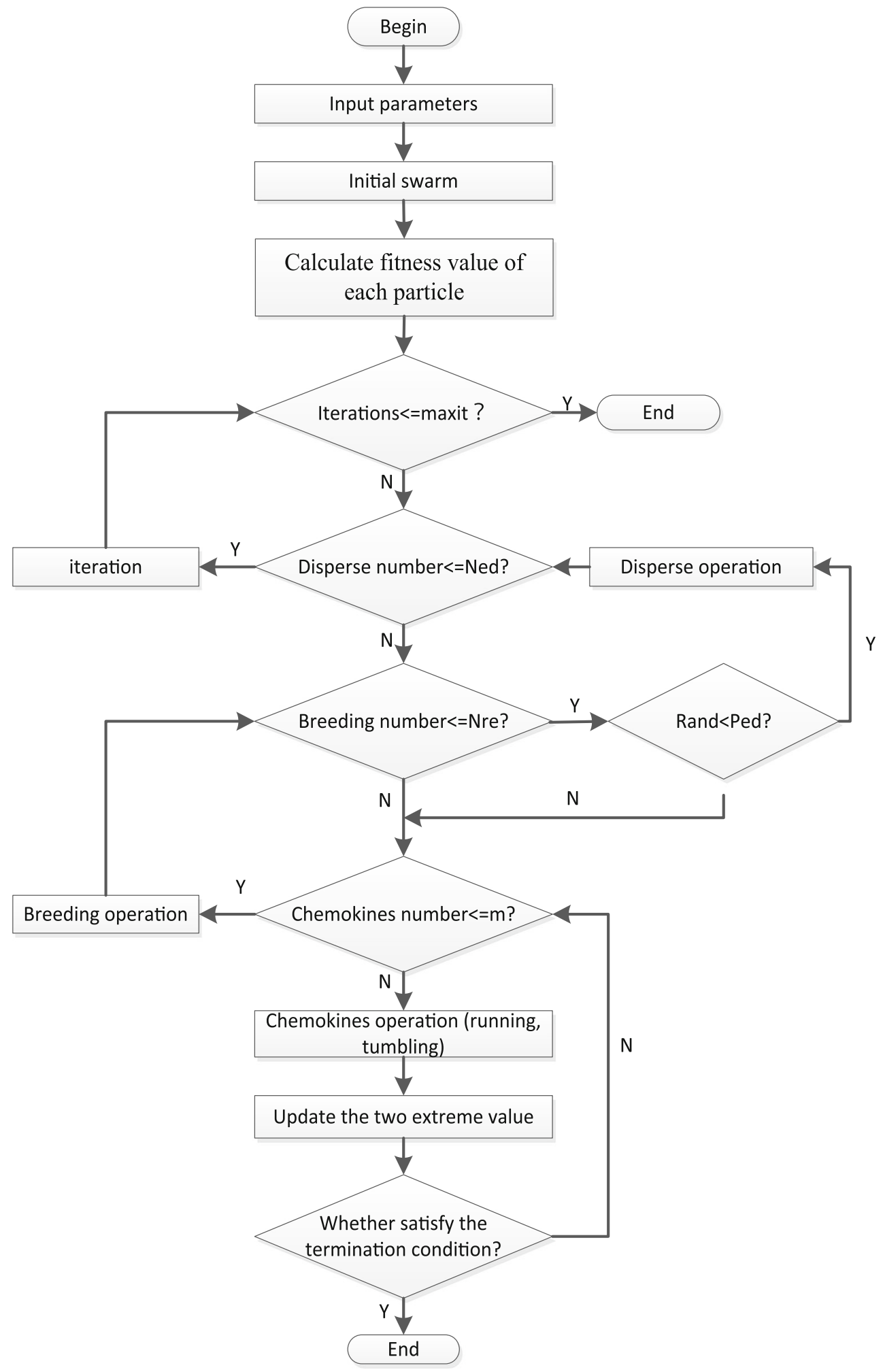

Fig. 1 The flowchart of the PSO-ABFA 
Photovoltaic arrays

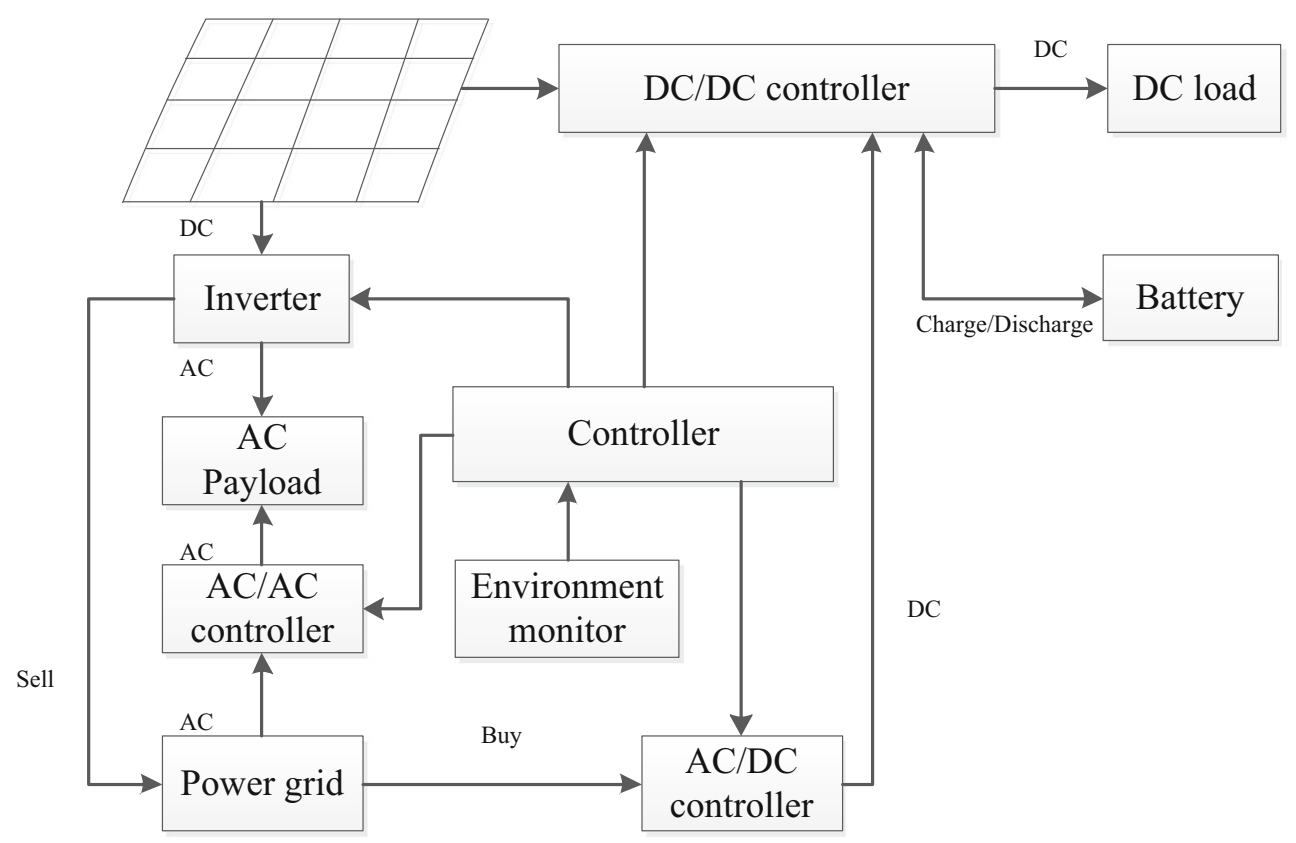

Fig. 2 The structure of the photovoltaic system

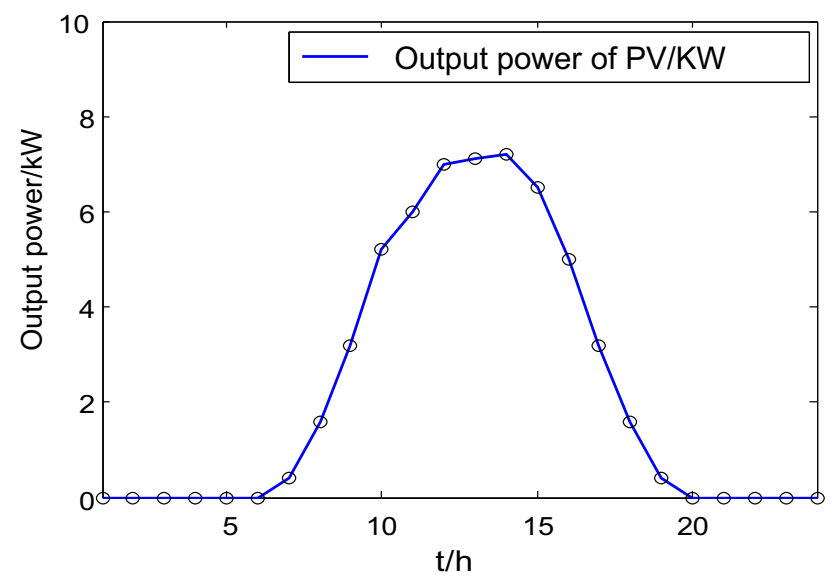

Fig. 3 Output power curve of the PV arrays

where the photovoltaic system ceases the sale of the electricity to the power grid. The battery achieves its maximum power at the end of this period after charging during the day. The following period between 18:00 and 21:00 is another peak period, where the battery is able to sell electricity to the power grid to generate income after the satisfaction of its own payload. The battery power reaches its minimal value at 21:00. 21:00 to 23:00 is another flat period, the battery power remains at its minimal value, and the photovoltaic system buys electricity from the power grid only for the payload.

Figures 6 and 7 show the SOC curve. It can be observed from Fig. 6 that the battery charges during the trough period and discharges during the peak period. Since the SOC is

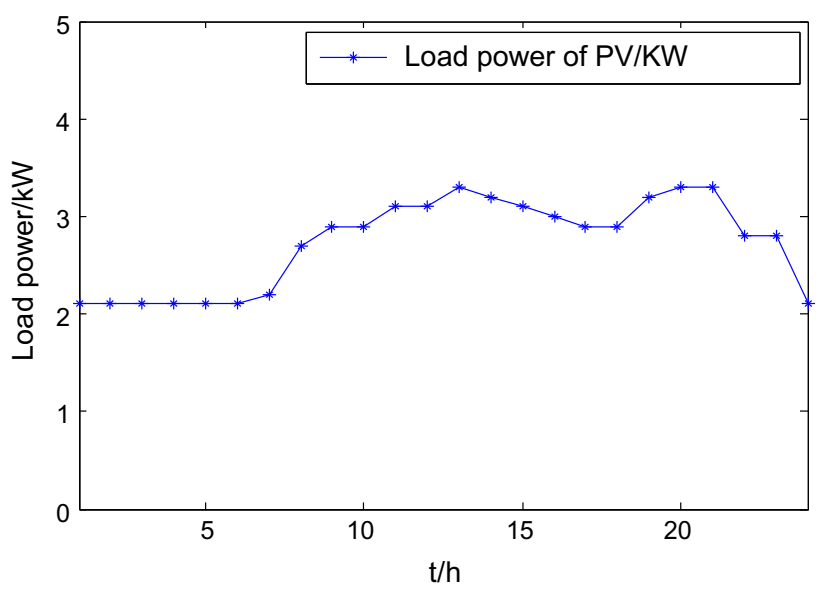

Fig. 4 Load curve of PV system

always controlled within an acceptable range during both the charging and discharging phases, usage and reliability of the battery have been guaranteed with an extended lifespan. It can be observed from Fig. 7 that the household photovoltaic system is buying electricity from and selling electricity to the power grid during the trough period and the peak period, respectively.

The daily operation profit before and after the optimization is listed in Table 2.

It is assume that the dispatching strategy of the household photovoltaic system is to sell all the generated power to the power grid, while simultaneously the payload consumes the 


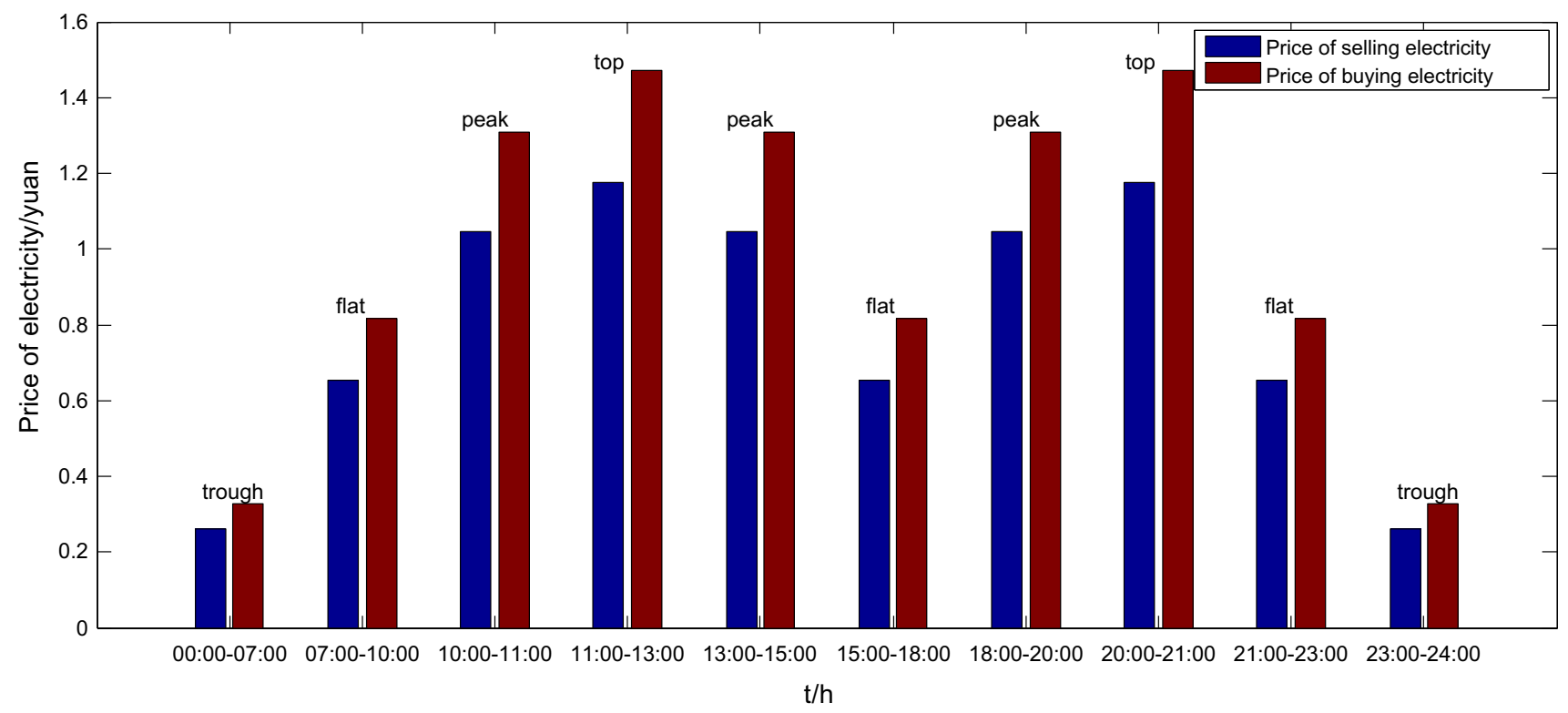

Fig. 5 Period division and the price of electricity

Table 1 The experiment optimization results

\begin{tabular}{|c|c|c|c|}
\hline$t / h$ & Battery power $(\mathrm{kW})$ & Selling power $(\mathrm{kW})$ & Buying power $(\mathrm{kW})$ \\
\hline 1 & -5 & 0 & 7.1000 \\
\hline 2 & -5 & 0 & 7.1000 \\
\hline 3 & -4.8088 & 0 & 6.9088 \\
\hline 4 & -3.8011 & 0 & 5.9011 \\
\hline 5 & -1.1392 & 0 & 3.2392 \\
\hline 6 & -0.2289 & 0 & 2.3289 \\
\hline 7 & -0.0214 & 0 & 1.8214 \\
\hline 8 & 0 & 0 & 1.1000 \\
\hline 9 & 0 & 0.3000 & 0 \\
\hline 10 & 0 & 2.3000 & 0 \\
\hline 11 & 5 & 7.9000 & 0 \\
\hline 12 & 5 & 8.9000 & 0 \\
\hline 13 & 5 & 8.8000 & 0 \\
\hline 14 & 4.3292 & 8.3292 & 0 \\
\hline 15 & 0.6641 & 4.0641 & 0 \\
\hline 16 & -5 & 0 & 3.0000 \\
\hline 17 & -5 & 0 & 4.7000 \\
\hline 18 & -5 & 0 & 6.3000 \\
\hline 19 & 5 & 2.2000 & 0 \\
\hline 20 & 5 & 1.7000 & 0 \\
\hline 21 & 5 & 1.7000 & 0 \\
\hline 22 & 0 & 0 & 2.8000 \\
\hline 23 & 0 & 0 & 2.8000 \\
\hline 24 & -0.0097 & 0 & 2.1097 \\
\hline
\end{tabular}

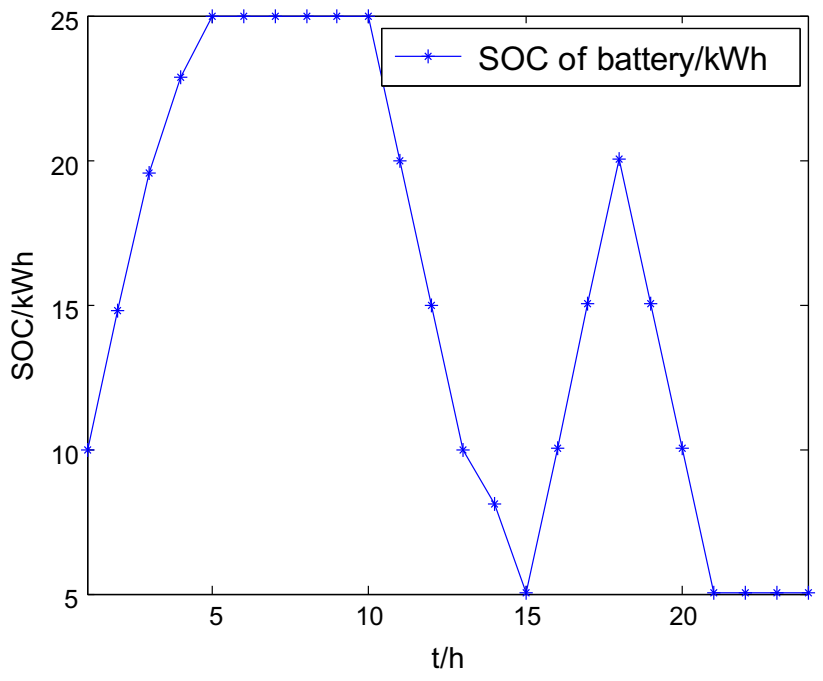

Fig. 6 SOC curve of battery

power bought from the power grid before the optimization. It is apparent that the daily operation profit after optimization is far better than those before optimization from the user benefit perspective.

The convergence curves of the three algorithms are shown in Fig. 8. It can be observed from Fig. 8 that the proposed APSO-BFA outperforms both PSO and PSO-BFA in terms of the convergence speed and accuracy due to the use of the chemokines, breeding, dispersion and self-adaptive step operations. 


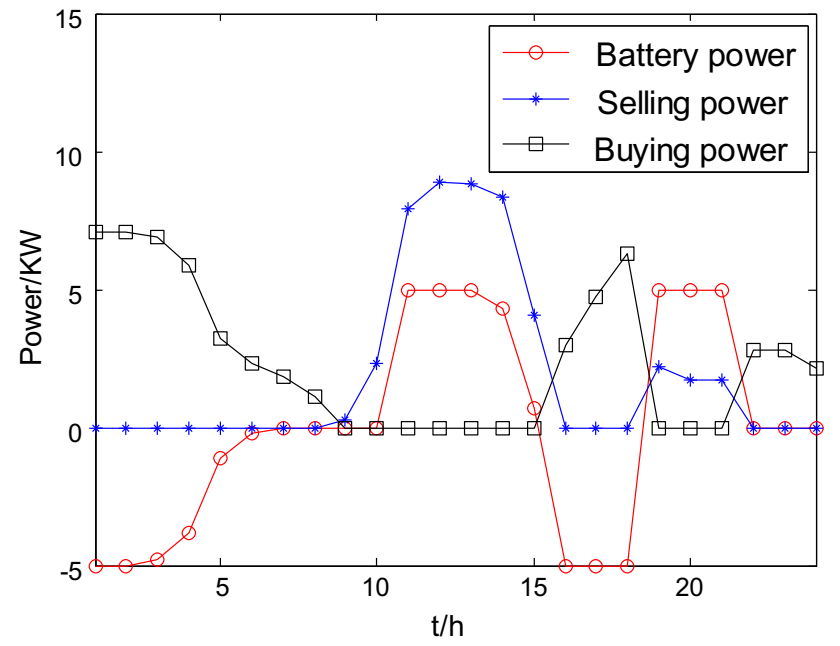

Fig. 7 The operation curve of PV system

Table 2 Profit comparison of different control strategies

\begin{tabular}{ll}
\hline Before optimization (RMB, yuan) & After optimization (RMB, yuan) \\
\hline-9.7222 & 16.6336
\end{tabular}

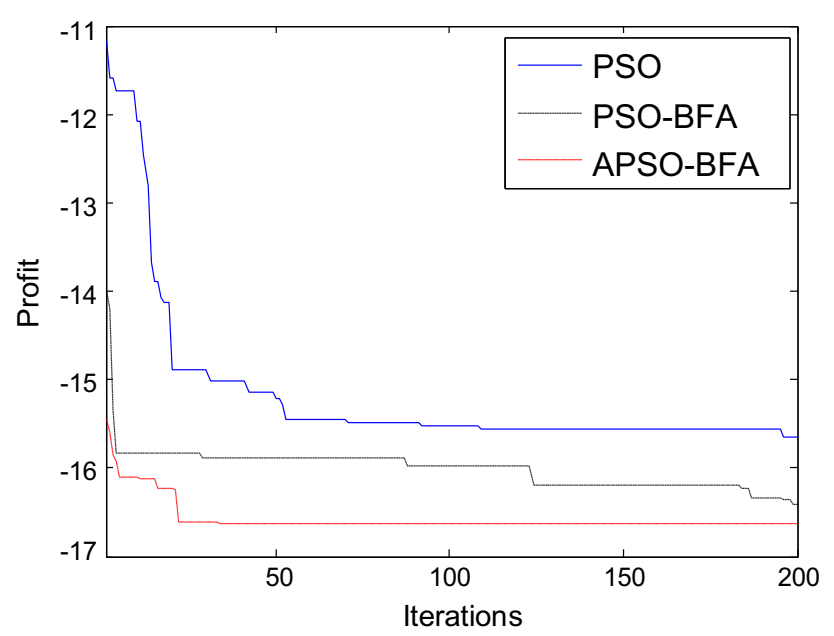

Fig. 8 Convergence curves of three algorithms

\section{Conclusions}

This paper proposes an ED model of photovoltaic system based on TOUEP to achieve a more rational efficient electricity provision and maximize economic benefit. The combination of APSO-BFA outperforms the previous algorithms, as the proposed algorithm benefits from a strong local searching capability and a high estimation accuracy using chemokines and breeding operations. The global searching capability is guaranteed via dispersing operation. Furthermore, the characteristics of fast convergence is achieved by an adaptive operation and the process of tracking two extreme values. Experiment data conducted based on TOUEP in Shandong Province demonstrate that the proposed model has the effect of peak cutting and valley filling by means of buying electricity when valley and selling it when peak which significantly enhances the stability of the power grid, as shown in Table 1. At the same time, the model gets an abundant profit after the optimization which is observed in Table 2. That is to say, the proposed strategy is able to provide the most economical electricity usage plan to users both in theory and practice. Whereas because of the limit of information resources, the ED model in this paper just applies to a specific season, more long-term research about different season is the target in the future research.

Open Access This article is distributed under the terms of the Creative Commons Attribution 4.0 International License (http://creativecomm ons.org/licenses/by/4.0/), which permits unrestricted use, distribution, and reproduction in any medium, provided you give appropriate credit to the original author(s) and the source, provide a link to the Creative Commons license, and indicate if changes were made.

\section{References}

1. Nandi, S.K.; Hoque, M.N.; Ghosh, H.R.; et al.: Assessment of wind and solar energy resources in Bangladesh. Arab. J. Sci. Eng. 38(11), 3113-3123 (2013)

2. Rabbani, M.; Ratlamwala, T.A.H.: Dincer I: Development of a new heliostat field-based integrated solar energy system for cogeneration. Arab. J. Sci. Eng. (2017). https://doi.org/10.1007/s13369017-2774-x

3. Yang, Z.X.: Research on Economic Operation Optimization of Hybrid Photovoltaic Systems Based on Time-of-Use Price, pp. 2-8. Shandong University, Jinan Shi (2013)

4. Juan, Z.; Zhongfu, T.; Qiang, L.: Analysis of time use power price in China. Mod. Electr. Power 22(2), 82-85 (2005)

5. Xin, A.; Cui, M.; Lei, Z.: Environmental and economic dispatch of Microgrid using chaotic ant swarm algorithms. J. North China Electr. Power Univ. 36(9), 1-6 (2009)

6. Jie, M.; Gengyin, L.: Dynamic economical dispatch for power system containing wind power and photovoltaic power. Power Syst. Clean Energy 29(11), 70-75 (2013)

7. Gc, L.I.A.O.: Solve environmental economic dispatch of Smart Microgrid containing distributed generation system - using chaotic quantum genetic algorithm. Int. J. Electr. Power Energy Syst. 43(1), 779-787 (2012)

8. Xi, Y.M.; Yao, W.W.; Hua, L.F.; Yuan, Z.S.: Optimal sizing of hybrid solar-wind distributed generation in an islanded micro grid using improved bacterial foraging algorithm. Proc. Chin. Soc. Electr. Eng. 31(25), 17-25 (2011)

9. Hongbin, W.; Xingyue, L.; Ming, D.: Dynamic economic dispatch of a microgrid: mathematical models and solution algorithm. Int. J. Electr. Power Energy Syst. 63(63), 336-346 (2014)

10. Jeyakumar, D.N.; Jayabarathi, T.; Raghunathan, T.: Particle swarm optimization for various types of economic dispatch problems. Int. J. Electr. Power Energy Syst. 28(1), 36-42 (2006)

11. Jiejin, C.; Xiaoqian, M.; Qiong, L.; et al.: A multi-objective chaotic particle swarm optimization for environmental/economic dispatch. Energy Convers. Manag. 50(5), 1318-1325 (2009) 
12. Chowdhury, A.; Zafar, H.; Panigrahi, B.K.; et al.: Dynamic economic dispatch using lbest-pso dynamically varying sub-swarms. Memet. Comput. 6(2), 85-95 (2014)

13. Guangyu, C.; Xiaoqun, D.: Optimal economic dispatch with valve loading effect using self-adaptive firefly algorithm. Appl. Intell. 42(2), 276-288 (2015)

14. Al-Awami, A.T.; Ansari, M.A.H.; Bennett, B.J.: Stochastic dynamic economic for grids with significant wind using mixed gaussian distribution. Arab. J. Sci. Eng. 41(2), 545-553 (2016)

15. Eberhart, R.; Kennedy, J.: A new optimizer using particle swarm theory. In: Proceedings of the Sixth International Symposium on Micro-Machine and Human Science (1995)

16. Kennedy, J.; Eberhart, R.: Particle swarm optimization. In: IEEE International Conference on Neural Networks, vol. 4, pp. 19421948 (2002)

17. Huang, J.H.; Su, S.B.; Ma, Y.; et al.: Particle swarm optimization algorithm with adaptive mutation. Comput. Eng. 34(16), 188-190 (2010)

18. Zhen, S.L.U.; Hou, Z.R.; Juan, D.U.: Particle swarm optimization with adaptive mutation. Front. Electr. Electron. Eng. 1(1), 99-104 (2006)
19. Saboori, H.; Hemmati, R.: Optimal management and planning of storage systems based on particle swarm optimization technique. J. Renew. Sustain. Energy 8(2), 453-475 (2016)

20. Tabet, I.; Touafek, K.; Bellel, N.; et al.: Optimization of angle of inclination of the hybrid photovoltaic-thermal solar collector using particle swarm optimization algorithm. J. Renew. Sustain. Energy 6(5), 191-224 (2014)

21. Liu, Y.; Passino, K.M.: Biomimicry of social foraging bacteria for distributed optimization: models, principles, and emergent behaviors. J. Optim. Theory Appl. 115(3), 603-628 (2002)

22. Dong, H.K.; Abraham, A.; Cho, J.H.: A hybrid genetic algorithm and bacterial foraging approach for global optimization. Inf. Sci. 177(18), 3918-3937 (2007)

23. Ying, C.; Hua, M.; Zhen, J.; Qing, H.W.: Fast bacterial swarming algorithm based on particle swarm optimization. J. Data Acquis. Process. 25(4), 442-448 (2010) 